\title{
Graphene Blisters with Switchable Shapes Controlled by Pressure and Adhesion
}

\author{
Narasimha G. Boddeti, ${ }^{\dagger}$ Xinghui Liu, ${ }^{\dagger}$ Rong Long, ${ }^{\dagger, \ddagger}$ Jianliang Xiao $^{\dagger}{ }^{\text {J. Scott Bunch, }}{ }^{\perp}$ \\ and Martin L. Dunn*,\$ \\ ${ }^{\dagger}$ Department of Mechanical Engineering, University of Colorado, Boulder, Colorado 80309, United States \\ ${ }^{\ddagger}$ Department of Mechanical Engineering, University of Alberta, Edmonton, Alberta T6G 2G8, Canada \\ ${ }^{\S}$ Singapore University of Technology and Design, Singapore, 138682 \\ ${ }^{\perp}$ Department of Mechanical Engineering, Boston University, Boston, Massachusetts 02215, United States
}

Supporting Information

ABSTRACT: We created graphene blisters that cover and seal an annular cylinder-shaped microcavity in $\mathrm{SiO}_{2}$ substrate filled with a gas. By controlling the pressure difference between the gas inside and outside of the microcavity, we switch the graphene membrane between multiple stable equilibrium configurations. We carried out experiments starting from the situation where the pressure of the gas inside and outside of the microcavity is set equal to a prescribed charging pressure, $p_{0}$ and the graphene membrane covers the cavity like an annular drum, adhered to the central post and the surrounding substrate due to van der Waals forces. We decrease the

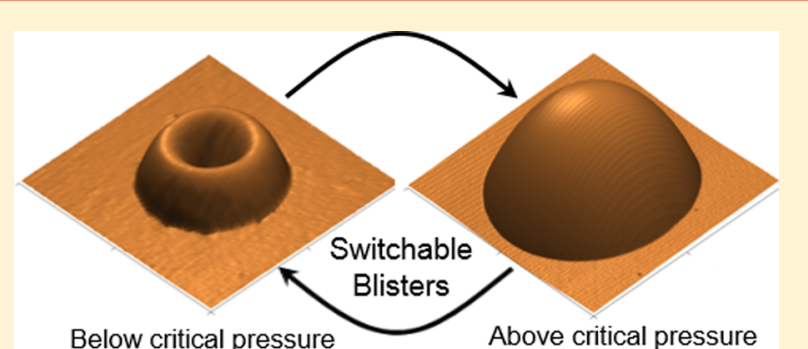
outside pressure to a value, $p_{\mathrm{e}}$ which causes it to bulge into an annular blister. We systematically increase the charging pressure by repeating this procedure causing the annular blister to continue to bulge until a critical charging pressure $p_{\mathrm{c}}^{\mathrm{i}}$ is reached. At this point the graphene membrane delaminates from the post in an unstable manner, resulting in a switch of graphene membrane shape from an annular to a spherical blister. Continued increase of the charging pressure results in the spherical blister growing with its height increasing, but maintaining a constant radius until a second critical charging pressure $p_{\mathrm{c}}^{\mathrm{o}}$ is reached at which point the blister begins to delaminate from the periphery of the cavity in a stable manner. Here, we report a series of experiments as well as a mechanics and thermodynamic model that demonstrate how the interplay among system parameters (geometry, graphene stiffness (number of layers), pressure, and adhesion energy) results in the ability to controllably switch graphene blisters among different shapes. Arrays of these blisters can be envisioned to create pressure-switchable surface properties where the difference between patterns of annular versus spherical blisters will impact functionalities such as wettability, friction, adhesion, and surface wave characteristics.

KEYWORDS: Graphene, annular blister, spherical blister, adhesion, switching, instability

G raphene is an atomically thin $2 \mathrm{D}$ material that can be

obtained via mechanical exfoliation from graphite $e^{1,2}$ and more recently it has been grown by chemical vapor deposition. $^{3-7}$ It can exist as a monolayer or as multiple layers that are bonded by van der Waals interactions between individual sheets. Graphene has attractive mechanical, electrical, thermal, and optical properties and has potential applications in wide-ranging fields. ${ }^{8,9}$ Though stiff (Young's modulus $\sim 1$ $\mathrm{TPa}^{10}$ ), being atomically thin, monolayer, and few layer graphene membranes are flexible in bending and can adhere to substrates or surrounding structures with high conformity. ${ }^{11-14}$ Despite, and partly because of, adhesion, graphene can form blisters of various shapes on the substrates due to unintentionally $^{15,16}$ or deliberately trapped gas, ${ }^{17,18}$ strain mismatch, ${ }^{19}$ and intercalated nanoparticles. ${ }^{20}$ Potential applications of these graphene blisters include a liquid cell to observe chemical reactions, ${ }^{21}$ an optical lens with adjustable focal length, ${ }^{16}$ and a means to strain engineer graphene electronic properites. ${ }^{22}$ In this paper, we describe a new type of graphene blister with switchable shapes controlled by pressure and adhesion. A similar geometry, but with a constant pressure loading, has been previously used to measure the mechanical and adhesive properties of soft films ${ }^{23,24}$ a few micrometers thick, as well as to observe pull-in instabilities in graphene membranes due to interfacial forces. ${ }^{25}$ Here we adopt this geometry and modify it by using a trapped gas with constant number of molecules (or equivalently fixed mass) instead of a constant pressure load, along the lines of a fixed mass blister test. ${ }^{18,26}$ We vary the pressure of the trapped gas (thereby varying the mass and number of molecules) to achieve switchable blister configurations and employ a nonlinear mechanics model in a thermodynamic framework to under-

Received: September 29, 2013

Revised: November 8, 2013

Published: November 13, 2013 

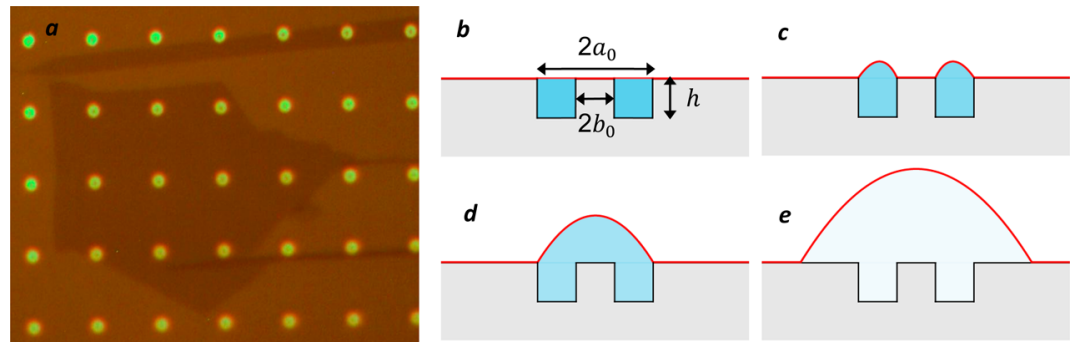

Figure 1. (a) Optical image of microcavities covered with a monolayer graphene membrane. Graphene blister switching process as the charging pressure is systematically increased: (b) a microcavity with pressurized gas molecules sealed by graphene; (c) as the gas expands isothermally, membrane deforms into an annular blister; (d) as the gas further expands, the membrane delaminates from the island forming a circular bulge; (e) finally, the membrane delaminates in the outward direction. The graphene membrane is depicted as a red line and the blue shaded region is the trapped gas.

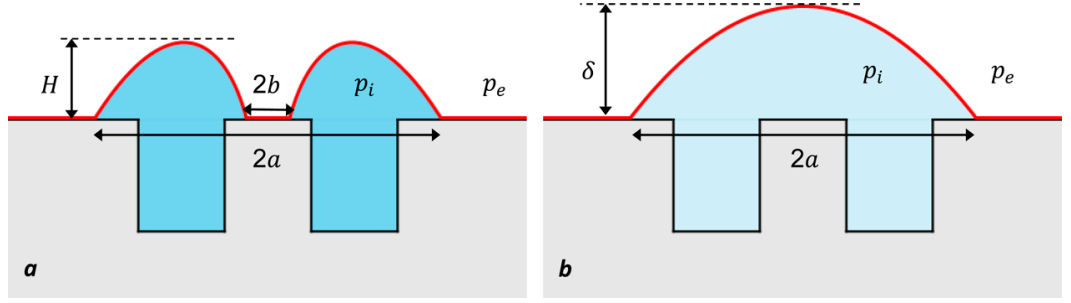

Figure 2. Schematics showing the most general form of the (a) annular blister and (b) spherical blister.

stand the interrelationship between the geometry, adhesion energy, and the pressure applied on the graphene membrane.

Our experimental system includes graphene flakes mechanically exfoliated on top of a lithographically patterned array of annular cylindrical cavities on a silicon chip with a $90 \mathrm{~nm}$ thick thermally grown oxide layer. We call each of the graphene sealed microcavities that trap gas due to the impermeable nature of the graphene membranes ${ }^{17}$ a device. We fabricated eight monolayered devices, the optical image of which is shown in Figure 1a, with outer radius $a_{0}=1.50 \mu \mathrm{m}$, inner radius $b_{0}=$ $0.35 \mu \mathrm{m}$, and depth $h=112 \mathrm{~nm}$ and eight five-layered devices with dimensions $a_{0}=1.70 \mu \mathrm{m}, b_{0}=0.25 \mu \mathrm{m}$, and $h=106.5 \mathrm{~nm}$. The thickness of the graphene membranes is verified optically as well as using Raman spectroscopy ${ }^{27}$ (see Supporting Information), which in principle could also be used to measure the strain in the deforming graphene. ${ }^{19,28,29}$ Initially, for each device the pressure inside the microcavity, $p_{\mathrm{i}}$ is the same as the pressure outside the cavity, $p_{\mathrm{e}}$ (Figure $1 \mathrm{~b}$ ). We pressurize the devices using a previously developed technique ${ }^{17,18}$ where they are put in a $\mathrm{N}_{2}$-containing pressure chamber at a prescribed pressure, $p_{0}$, which we call the charging pressure, and left long enough ( $\sim 7$ days $)$ such that the $\mathrm{N}_{2}$ molecules diffuse through the oxide layer and equilibrate the pressures inside and outside the cavity to the charging pressure, that is, $p_{\mathrm{i}}=p_{\mathrm{e}}=p_{0}$. We then remove the devices from the pressure chamber, which reduces the pressure outside of the cavity from $p_{0}$ to the ambient pressure, $p_{\mathrm{e}}\left(p_{\mathrm{e}}<p_{\mathrm{i}}<p_{0}\right)$ and establishes a pressure difference across the suspended graphene membrane that causes it to bulge into an annular blister (Figure 1c). We measure the deformed shape of the graphene membrane using an atomic force microscope (AFM). As we systematically increased the charging pressure, the graphene membranes delaminated from the island to form spherical cap-shaped bulges (Figure 1d) and then as the charging pressure increased further, the membranes delaminated in a stable manner from the outer boundary as shown in Figure 1e. Hence, with increasing charging pressure we achieved graphene blisters with switchable shapes (annular and circular) and size (height and radius) involving transitions from (i) an annular blister with fixed radii to (ii) a spherical blister with a fixed radius to (iii) a spherical blister with an increasing radius. Similar switching transitions, in the reverse order can be obtained by decreasing the pressure although hysteretic behavior is expected for the spherical to annular transition. ${ }^{25}$ We denote the charging pressures at which these transitions occur by $p_{\mathrm{c}}^{\mathrm{i}}$ and $p_{\mathrm{c}}^{\mathrm{o}}$, respectively. To understand the behavior of the switchable blister we analyzed the mechanics as well as thermodynamics involved in the experiment, including the stability of delamination from the island and the outer boundary.

At a given pressure the graphene membrane deforms into either an annular or a spherical cap shape as shown in Figure 2. The equilibrium configuration for the annular shape can be any of the following situations ( $a$ and $b$ here are the outer and inner radii of the general annular blister shown in Figure 2a):

1. $a=a_{0}, b=b_{0}$ : the membrane did not delaminate from either the island/post or at the outer boundary.

2. $a>a_{0}, b=b_{0}$ : the membrane delaminated at the outer boundary but not from the island.

3. $a=a_{0}, b>b_{0}$ : the membrane delaminated from the island but not at the outer boundary.

4. $a>a_{0}, b>b_{0}$ : the membrane delaminated both from the island and at the outer boundary.

Two configurations are possible when the membrane delaminates from the island and deforms into a spherical cap:

1. $a=a_{0}$ : no delamination from the edge of the outer boundary.

2. $a>a_{0}$ : delamination at the outer boundary.

To analyze the deformation and delamination mechanics of the graphene membranes observed in the experiment, we consider the experimental system consisting of the graphene membrane adhered to the substrate, the graphene sealed microcavity with pressurized gas trapped inside and the ambient air outside to be a thermodynamic system with free energy, $F$. We modeled the trapped gas inside the microcavity 
as an ideal gas that can expand isothermally, the graphene membrane as an isotropic elastic membrane (Young's Modulus $E$, Poisson's ratio $\nu$ and thickness $t$ ), and the graphenesubstrate adhesive interactions are assumed to be characterized by a uniform adhesion energy, $\Gamma$. Expressing the free energy $F$ as a function of the system parameters and minimizing it then provides the equilibrium configuration observed in the experiment.

For annular-shaped blisters, we developed an approximate solution based on the approaches of Saif et al. and Williams. ${ }^{30,31}$ We assume that the tangential strain in the deformed membrane is negligible compared to the radial strain, and that the radial tension in the membrane is uniform. This uniform radial tension, denoted by $S$, is obtained by averaging the radial strain over the area of the deformed region. This approximate analysis provides relations that describe the deformed configurations

$$
\begin{gathered}
H=\frac{p}{4 S}\left(a^{2}-\frac{r_{0}^{2}}{2}+\frac{r_{0}^{2}}{2} \ln \left[\frac{r_{0}^{2}}{2 a^{2}}\right]\right) \\
V_{a}=\frac{\pi}{2} \frac{p}{4 S}\left(a^{2}-b^{2}\right)\left(a^{2}+b^{2}-r_{0}^{2}\right)
\end{gathered}
$$

Here $r_{0}=\left(a^{2}-b^{2}\right) /(\ln [\mathrm{a} / \mathrm{b}])$ and $S=\left\{\left[\left(E t p^{2}\right) /(16(1-\right.\right.$ $\left.\left.\left.\left.\nu^{2}\right)\right)\right]\left(a^{2}+b^{2}-r_{0}^{2}\right)\right\}^{1 / 3}$. $H$ denotes the maximum deflection as shown in Figure 2a, which occurs at $r=r_{0} / \sqrt{2}, p$ is the pressure difference across the membrane, and $V_{\mathrm{a}}$ is the volume displaced by the deformed membrane. In both the annular and spherical deformation cases, the pressure loads applied during our experiments are large enough that we can neglect the effects of the initial tension (usually on the order of $0.1 \mathrm{~N} / \mathrm{m}^{32,33}$ ) on the mechanics of the graphene membrane. If needed, the effect of initial tension on the mechanics can be readily included. Details regarding both the approximate analysis and the finite element simulations are presented in the Supporting Information.

When there is no delamination from the substrate, either inward from the post or outward from the cavity boundary, the mechanics of the membrane are completely described by the relations in eq 1 when coupled with ideal gas equation $p_{\mathrm{i}}\left(V_{0}+\right.$ $\left.V_{\mathrm{a}}\right)=p_{0} V_{0}$ under isothermal conditions. These two equations provide a relation between known parameters $a=a_{0}, b=b_{0}, E t$, $\nu, p_{0}, p_{\mathrm{e}}$ and the equilibrium pressure in the cavity $p_{\mathrm{i}}$

$$
\left(\frac{p_{0}-p_{\mathrm{i}}}{p_{\mathrm{i}}}\right)^{3} V_{0}^{3}=\frac{\pi^{3}\left(1-\nu^{2}\right)}{32 E t}\left(a^{2}-b^{2}\right)^{3}\left(a^{2}+b^{2}-r_{0}^{2}\right)^{2}\left(p_{\mathrm{i}}-p_{\mathrm{e}}\right)
$$

Once the equilibrium pressure $p_{\mathrm{i}}$ is determined, we can obtain the maximum deflection $H$ that characterizes the deformation using eq 1 .

If the membrane delaminates from the substrate, the radii $a$ and $b$ in the most general annular blister shape (Figure $2 a$ ) are unknown and eq 2 is insufficient to determine the equilibrium configuration. To determine the system behavior in this case we formulate the free energy, $F$ which includes contributions from the strain energy stored in the deforming membrane, the free energy associated with isothermal expansion of the $N$ trapped gas molecules in the chamber, the adhesion energy, and the free energy change of the external environment that is held at a pressure $p_{\mathrm{e}}$. The free energy (details in Supporting Information) can then be written in terms of the independent variables $a$ and $b$

$$
\begin{aligned}
& F(a, b)=\frac{\left(p_{\mathrm{i}}-p_{\mathrm{e}}\right) V_{\mathrm{a}}}{4}-p_{0} V_{0} \ln \left[\frac{V_{0}+V_{\mathrm{a}}}{V_{0}}\right]+p_{\mathrm{e}} V_{\mathrm{a}} \\
& \quad+\Gamma \pi\left(a^{2}-a_{0}^{2}\right)+\Gamma \pi\left(b_{0}^{2}-b^{2}\right)
\end{aligned}
$$

If delamination occurs in both the inward and outward directions without completely peeling from the island, that is, $0<b<b_{0}$, then the equilibrium configuration is found by minimizing $F(a, b)$ with respect to $a$ and $b$ to obtain two simultaneous relations among $\Gamma, a, b$, and $p$

$$
\begin{aligned}
& \Gamma=\frac{p}{8}\left(\frac{p\left(1-\nu^{2}\right)}{4 E t}\right)^{1 / 3} \frac{5 a^{4}+a^{2} b^{2}-5 a^{2} r_{0}^{2}+r_{0}^{4}}{a^{2}\left(a^{2}+b^{2}-r_{0}^{2}\right)^{1 / 3}} \\
& \Gamma=\frac{p}{8}\left(\frac{p\left(1-\nu^{2}\right)}{4 E t}\right)^{1 / 3} \frac{5 b^{4}+a^{2} b^{2}-5 b^{2} r_{0}^{2}+r_{0}^{4}}{b^{2}\left(a^{2}+b^{2}-r_{0}^{2}\right)^{1 / 3}}
\end{aligned}
$$

Here $p=p_{\mathrm{i}}-p_{\mathrm{e}}$ is obtained from eq 2. Equation 4 dictates that simultaneous delamination from the island and the outer boundary is not possible in general when the adhesion energies are the same on the island and on the outer boundary. Hence we look at $F\left(a_{0}, b\right)$ and $F\left(a, b_{0}\right)$ that denote free energies when delamination occurs exclusively inward or outward, respectively. Equation 4 then shows that the pressure required to initiate delamination in the outward direction is greater than that for the inward direction. This is due to the differences in the magnitude and sign of the delamination front curvature and is consistent with our experimental observations where we see delamination from the island first and then the outer boundary. Therefore, the critical charging pressure $p_{c}^{\mathrm{i}}$ at which the membrane delaminates from the island is obtained by putting $a$ $=a_{0}$ and $b=b_{0}$ in the second relation in eq 4 and combining the result with the ideal gas equation

$$
p_{\mathrm{c}}^{\mathrm{i}}=\left(\left(8 \Gamma\left(\frac{4 E t}{1-\nu^{2}}\right)^{1 / 3} \frac{b_{0}^{2}\left(a_{0}^{2}+b_{0}^{2}-r_{0}^{2}\right)^{1 / 3}}{5 b_{0}^{4}+a_{0}^{2} b_{0}^{2}-5 b_{0}^{2} r_{0}^{2}+r_{0}^{4}}\right)^{3 / 4}+p_{\mathrm{e}}\right)\left(\frac{V_{0}+V_{a}\left(a_{0}\right)}{V_{0}}\right)
$$

Using the same argument as above, it can be shown that the membrane delaminates from the island preferably if the adhesion energy on the island is lower than that on the outer boundary. However, if the adhesion energy on the island is larger than that on the outer perimeter, then the membrane could preferentially delaminate from the outer boundary (see Supporting Information). It is also found that the stability of the delamination depends on the initial volume, $V_{0}$. For initial volumes smaller than a critical value which depends on the cavity geometry, membrane elastic properties, and adhesion energy, the delamination can be made stable; but for initial volumes larger than the critical value it is always unstable leading to complete delamination from the island (see Supporting Information for an example).

The free energy of the spherical blister (Figure 2b), which is formed when the membrane delaminates from the island completely, is similar to the annular blister free energy except that now instead of eq 1 we use the well-known Hencky's solution. ${ }^{26,30,34}$ This analysis gives us a relation between $\Gamma, \delta$, $p$, and $a$ given the other system parameters. ${ }^{35}$

$$
\Gamma=\frac{5 C_{1}}{4}\left(\frac{p_{0} V_{0}}{V_{0}+V_{s}(a)}-p_{\mathrm{e}}\right) \delta(a)=\frac{5 C_{1} C_{2}}{4}\left(\frac{p(a)^{4} a^{4}}{E t}\right)^{1 / 3}
$$



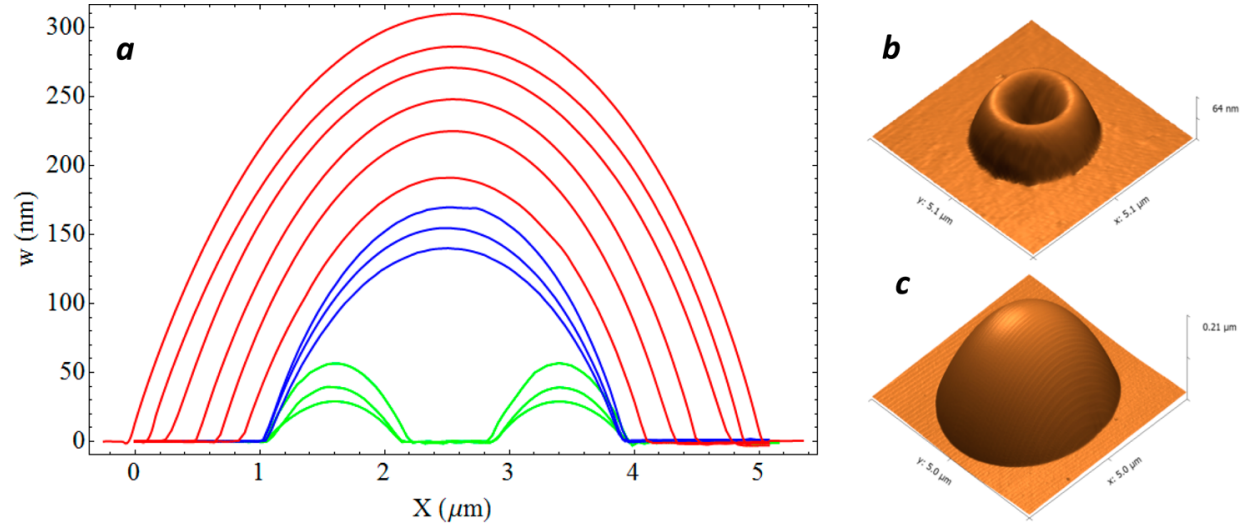

Figure 3. (a) Cross sections of AFM height images for monolayered graphene blisters: green (annular), blue (spherical with no delamination), and red (spherical with delamination). The pressures at which they are obtained in increasing order are $p_{0}=289.8,512.6,733.0,929.0,1223.0,1659.0$, 2051.0, 2557.0, 3010.0, 3431.0, 3755.0, and $4165.0 \mathrm{kPa}$. (b, c) Three-dimensional rendering of annular and spherical blisters obtained by AFM respectively.
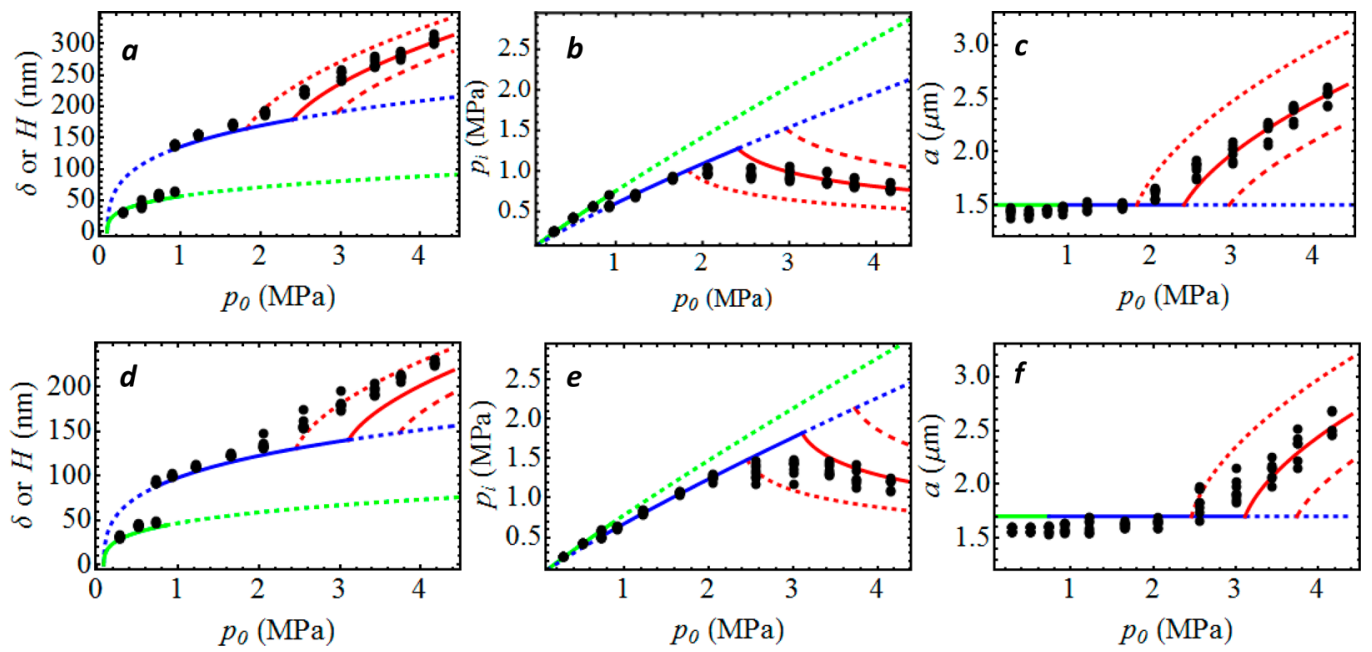

Figure 4. (a,d) Maximum deflection ( $\delta=$ spherical, $H=$ annular); (b,e) equilibrium pressure in the microcavity $\left(p_{\mathrm{i}}\right)$; and $(\mathrm{c}, \mathrm{f})$ outer radius of the circular bulge $(a)$ versus the charging pressure $\left(p_{0}\right)$. Panels $(a-c)$ are for graphene monolayers and $(\mathrm{d}-\mathrm{f})$ are for multilayers. In each case, the symbols are measurements and the curves are theory. The green curve is for the annular deformation, the blue curve for the spherical deformation without delamination, and the red curves are for spherical deformation with delamination for different values of adhesion energies (dashed, 100 (120) $\mathrm{mJ} / \mathrm{m}^{2}$, solid, $140(160) \mathrm{mJ} / \mathrm{m}^{2}$, and long dashed, $180(200) \mathrm{mJ} / \mathrm{m}^{2}$ for monolayer and multilayer membranes).

Here, $\delta$ is the maximum deflection of the membrane which occurs at $r=0$ and $C_{1}$ and $C_{2}$ are constants obtained from Hencky's analysis and they depend on Poisson's ratio. Unlike the case of annular blister delamination, spherical blister delamination is stable. ${ }^{18,26}$ Substituting $a=a_{0}$ in eq 6 yields the critical charging pressure for outward delamination, $p_{c}^{o}$ for a given value of $\Gamma$. Thus, eqs 5 and 6 show that the charging pressures at which switching occurs, $p_{\mathrm{c}}^{\mathrm{i}}$ and $p_{\mathrm{c}}^{\mathrm{o}}$ are functions of the cavity geometry $\left(a_{0}, b_{0}\right.$, and $\left.V_{0}\right)$, mechanical (Et and $\nu$ ), and adhesion $(\Gamma)$ properties. We can use eq 6 with measured $(a, \delta)$ pairs to determine the adhesion energy $\Gamma$. Additionally, the analytical form of our analysis makes it easy to use to create phase diagrams of system parameters as a function of charging pressure.

Full-field measurements of deformed blisters show that the deformations are axisymmetric (see Supporting Information for full AFM images), allowing us to describe the deformed configurations using just the deflection along a diametrical chord. Measured deflection profiles at different charging pressures for a representative device are plotted in Figure 3. At charging pressures below $750 \mathrm{kPa}$ for this particular device, (green curves), the membrane is adhered to the island, and as we gradually increase the charging pressure it delaminates from the island (at $929 \mathrm{kPa}$ ). At even higher pressures (about $2 \mathrm{MPa}$, red curves), the membrane then starts delaminating in the outward direction. Also shown in Figure 3 are full-field 3D AFM height scans of an annular and a spherical blister, demonstrating the axisymmetric deformation.

Figure $4 \mathrm{a}-\mathrm{c}$ show the experimentally determined maximum deflection $(H$ or $\delta)$, the internal cavity pressure $\left(p_{\mathrm{i}}\right)$ and the blister outer radius $(a)$ for the monolayer devices as a function of charging pressure $\left(p_{\mathrm{o}}\right)$. Figure $4 \mathrm{~d}-\mathrm{f}$ show the same quantities for the five-layer devices. Theoretical estimates are also shown in each figure. Both experiments and theory show three configurations: (i) annular blisters (green lines), (ii) spherical blister before delamination (blue lines), and (iii) spherical blisters after delamination from the outer boundary (red lines).

In Figure $4 a-c$, the solid, dashed, and long-dashed red curves are calculated with different values of adhesion energy that are $140 \pm 40 \mathrm{~mJ} / \mathrm{m}^{2}$ where $140 \mathrm{~mJ} / \mathrm{m}^{2}$ is the average adhesion energy for monolayer membranes obtained by applying eq 6 
(with $E t=340 \mathrm{~N} / \mathrm{m}$ and $\nu=0.16,{ }^{36} C_{1}=0.525, C_{2}=0.686$ ) to the last four data points where significant outward delamination occurs and thus our model is expected to be most accurate (complete details are given in the Supporting Information). It is noteworthy that these adhesion energies are lower than our previously reported range, $310-450 \mathrm{~mJ} / \mathrm{m}^{2}$ with the same substrate material. ${ }^{18}$ We do not fully understand the reasons for these differences but note that the fabrication process details were different here, and it would not be surprising that this lead to different surface conditions and thus adhesion energy. Our emphasis here is on the development of the switchable graphene blisters and understanding their behavior, and so the details of the adhesion energy are not essential to this study, only that we can measure it. To this end, our measurements for monolayered devices are self-consistent among $H$ or $\delta, p_{\mathrm{i}}$, and $a$ and show good agreement with the theory.

Corresponding results for the five-layer devices shown in Figure $4 \mathrm{~d}-\mathrm{f}$ are similar qualitatively but differ from the monolayers in two ways. First, the best-fit value of adhesion energy is $160 \mathrm{~mJ} / \mathrm{m}^{2}$; we think the difference in adhesion energies for monolayer and five-layers may result from the complicated dependence of adhesion energy on the number of layers, surface topography, and interfacial forces. ${ }^{37-39}$ Second, the behavior is not as self-consistent among $H$ or $\delta, p_{\mathrm{i}}$, and $a$; while the agreement between experiment and theory is reasonable, it is not as good as that for the monolayer. We attribute this to wrinkling that appears to occur during deformation of the multilayer devices (Supporting Information, Figure 13). While the broad nature of the axisymmetric deformation exists, the wrinkling indicates that our analytical model is not likely to work as well. Physically, we think the wrinkling is facilitated by sliding of the membrane near the perimeter of the blister. We can approximate the effect of sliding by relaxing our assumption that the membranes are clamped by the adhesive interactions at the boundaries. Instead, if we assume that the membranes are constrained from vertical movement but allowed to slide on the substrate then the membrane behaves softer than an equivalent clamped membrane with no sliding. ${ }^{40}$ Incorporating the sliding boundary condition into our analysis results in good agreement between theory and experiment that is also self-consistent among $H$ or $\delta, p_{\mathrm{i}}$, and $a$ (Supporting Information, Figure 12). Using our best estimate for the adhesion energy near the edges of the cavity (both the pillar and the outer perimeter) leads to an estimate of $0.99(0.75) \mathrm{MPa}$ for the critical island delamination charging pressure, $p_{\mathrm{c}}^{\mathrm{i}}$ for monolayers (multilayers), which is in reasonable agreement with experimental values of $0.90(0.70) \mathrm{MPa}$. Likewise our theory provides an estimate of the critical charging pressure, $p_{\mathrm{c}}^{\mathrm{o}}$ of $2.0(2.1) \mathrm{MPa}$ for monolayered (multilayered) devices, again in reasonable agreement with experiment as seen in Figure 4.

In conclusion, we fabricated and demonstrated the operation of graphene membranes suspended on annular cylindrical cavities that form blisters with shapes that can be switched and tuned by the combination of pressure and adhesion. Our measurements showed that graphene membranes can be switched from an annular to a spherical shape with varying lateral size using the pressure exerted by a fixed mass of trapped gas. We modeled the experimental system using a new nonlinear membrane model (for the annular blister) and ideal gas behavior in a thermodynamic framework to determine the deformation characteristics and the critical charging pressures at which the blisters switch shapes as a function of system parameters (geometry, elastic properties, pressure, and adhesion energy). This ability of graphene blisters to switch configurations can be potentially used to create surfaces with tunable topography when covered with a patterned array of these devices. The devices can potentially be made individually addressable, thereby making the tuning process dynamic. Such devices with dynamically tunable topography can be used to make smart surfaces that can change their surface/interfacial properties, for instance wettability. This device geometry can be extended to making electromechanical devices where electrostatic force between the graphene membrane and the substrate can be used to control the switching while keeping the pressure inside the cavity fixed for improved control and faster operation.

\section{ASSOCIATED CONTENT}

\section{Supporting Information}

Device fabrication, determination of graphene thickness, annular blister mechanics, finite element simulations, free energy contributions, stability of the constant $\mathrm{N}$ island blister growth, AFM height scans of a device, sliding of graphene membranes, and adhesion energy and critical charging pressures. This material is available free of charge via the Internet at http://pubs.acs.org.

\section{AUTHOR INFORMATION}

\section{Corresponding Author}

*E-mail: martin_dunn@sutd.edu.sg.

\section{Notes}

The authors declare no competing financial interest.

\section{ACKNOWLEDGMENTS}

We thank Rishi Raj for the use of the Raman microscope. This work was supported by NSF Grants CMMI 0900832, CMMI Career 105440, the DARPA Center on Nanoscale Science and Technology for Integrated Micro/Nano-Electromechanical Transducers (iMINT), and in part by the NNIN and the National Science Foundation under Grant ECS-0335765.

\section{REFERENCES}

(1) Novoselov, K. S.; Geim, A. K.; Morozov, S. V; Jiang, D.; Zhang, Y.; Dubonos, S. V; Grigorieva, I. V; Firsov, A. A. Science 2004, 306, 666-9.

(2) Novoselov, K. S.; Jiang, D.; Schedin, F.; Booth, T. J.; Khotkevich, V. V; Morozov, S. V; Geim, A. K. Proc. Natl. Acad. Sci. U.S.A. 2005 , 102, 10451-3.

(3) Yu, Q.; Lian, J.; Siriponglert, S.; Li, H.; Chen, Y. P.; Pei, S.-S. Appl. Phys. Lett. 2008, 93, 113103.

(4) De Arco, L. G.; Kumar, A. IEEE Trans. Nanotechnol. 2009, 8, 135-138.

(5) Reina, A.; Jia, X.; Ho, J.; Nezich, D.; Son, H.; Bulovic, V.; Dresselhaus, M. S.; Kong, J. Nano Lett. 2009, 9, 30-5.

(6) Kim, K. S.; Zhao, Y.; Jang, H.; Lee, S. Y.; Kim, J. M.; Kim, K. S.; Ahn, J.-H.; Kim, P.; Choi, J.-Y.; Hong, B. H. Nature 2009, 457, 70610.

(7) Li, X.; Cai, W.; An, J.; Kim, S.; Nah, J.; Yang, D.; Piner, R.; Velamakanni, A.; Jung, I.; Tutuc, E.; Banerjee, S. K.; Colombo, L.; Ruoff, R. S. Science 2009, 324, 1312-4.

(8) Geim, A. K. Science 2009, 324, 1530-4.

(9) Novoselov, K. S.; Fal'ko, V. I.; Colombo, L.; Gellert, P. R.; Schwab, M. G.; Kim, K. Nature 2012, 490, 192-200.

(10) Lee, C.; Wei, X.; Kysar, J. W.; Hone, J. Science 2008, 321, 385- 
(11) Lui, C. H.; Liu, L.; Mak, K. F.; Flynn, G. W.; Heinz, T. F. Nature 2009, 462, 339-41.

(12) Cullen, W. G.; Yamamoto, M.; Burson, K. M.; Chen, J. H.; Jang, C.; Li, L.; Fuhrer, M. S.; Williams, E. D. Phys. Rev. Lett. 2010, 105, 215504.

(13) Low, T.; Perebeinos, V.; Tersoff, J.; Avouris, P. Phys. Rev. Lett. 2012, 108, 096601.

(14) Scharfenberg, S.; Mansukhani, N.; Chialvo, C.; Weaver, R. L.; Mason, N. Appl. Phys. Lett. 2012, 100, 021910.

(15) Stolyarova, E.; Stolyarov, D.; Bolotin, K.; Ryu, S.; Liu, L.; Rim, K. T.; Klima, M.; Hybertsen, M.; Pogorelsky, I.; Pavlishin, I.; Kusche, K.; Hone, J.; Kim, P.; Stormer, H. L.; Yakimenko, V.; Flynn, G. Nano Lett. 2009, 9, 332-7.

(16) Georgiou, T.; Britnell, L.; Blake, P.; Gorbachev, R. V.; Gholinia, A.; Geim, A. K.; Casiraghi, C.; Novoselov, K. S. Appl. Phys. Lett. 2011, 99, 093103.

(17) Bunch, J. S.; Verbridge, S. S.; Alden, J. S.; van der Zande, A. M.; Parpia, J. M.; Craighead, H. G.; McEuen, P. L. Nano Lett. 2008, 8, 2458-62.

(18) Koenig, S. P.; Boddeti, N. G.; Dunn, M. L.; Bunch, J. S. Nat. Nanotechnol. 2011, 6, 543-546.

(19) Pan, W.; Xiao, J.; Zhu, J.; Yu, C.; Zhang, G.; Ni, Z.; Watanabe, K.; Taniguchi, T.; Shi, Y.; Wang, X. Sci. Rep. 2012, 2, 893.

(20) Zong, Z.; Chen, C.-L.; Dokmeci, M. R.; Wan, K. J. Appl. Phys. 2010, 107, 026104.

(21) Yuk, J. M.; Park, J.; Ercius, P.; Kim, K.; Hellebusch, D. J.; Crommie, M. F.; Lee, J. Y.; Zettl, A.; Alivisatos, A. P. Science 2012, 336, 61-4.

(22) Levy, N.; Burke, S. A.; Meaker, K. L.; Panlasigui, M.; Zettl, A.; Guinea, F.; Castro Neto, A. H.; Crommie, M. F. Science 2010, 329, 544-7.

(23) Allen, M. G.; Senturia, S. D. J. Adhes. 1988, 25, 303-315.

(24) Allen, M. G.; Senturia, S. D. J. Adhes. 1989, 29, 219-231.

(25) Liu, X.; Boddeti, N. G.; Szpunar, M. R.; Wang, L.; Rodriguez, M. A.; Long, R.; Xiao, J.; Dunn, M. L.; Bunch, J. S. Nano Lett. 2013, 13 (5), 2309-2313.

(26) Wan, K.-T.; Mai, Y.-W. Acta Metall. Mater. 1995, 43, 41094115.

(27) Koh, Y. K.; Bae, M.-H.; Cahill, D. G.; Pop, E. ACS Nano 2011, $5,269-74$.

(28) Zabel, J.; Nair, R. R.; Ott, A.; Georgiou, T.; Geim, A. K.; Novoselov, K. S.; Casiraghi, C. Nano Lett. 2012, 12, 617-21.

(29) Kitt, A.; Qi, Z.; Remi, S.; Park, H. S.; Swan, A. K.; Goldberg, B. Nano Lett 2013, 13 (6), 2605-2610.

(30) Williams, J. G. Int. J. Fract. 1997, 87, 265-288.

(31) Saif, M. T. A.; Alaca, B. E.; Sehitoglu, H. J. Microelectromech. Syst. 1999, 8, 335-345.

(32) Barton, R. A.; Ilic, B.; van der Zande, A. M.; Whitney, W. S.; McEuen, P. L.; Parpia, J. M.; Craighead, H. G. Nano Lett. 2011, 11, 1232-6.

(33) Wang, L.; Travis, J. J.; Cavanagh, A. S.; Liu, X.; Koenig, S. P.; Huang, P. Y.; George, S. M.; Bunch, J. S. Nano Lett. 2012, 12, 370610.

(34) Hencky, H. Z. Math. Phys. 1915, 63, 311-317.

(35) Boddeti, N. G.; Koenig, S. P.; Long, R.; Xiao, J.; Bunch, J. S.; Dunn, M. L. J. Appl. Mech. 2013, 80 (4), 040909-.

(36) Blakslee, O. L. J. Appl. Phys. 1970, 41, 3373.

(37) Li, T.; Zhang, Z. J. Phys. D: Appl. Phys. 2010, 43, 075303.

(38) Gao, W.; Huang, R. J. Phys. D: Appl. Phys. 2011, 44, 452001.

(39) Viola Kusminskiy, S.; Campbell, D.; Castro Neto, A.; Guinea, F. Phys. Rev. B 2011, 83, 165405.

(40) Timoshenko, S.; Woinowsky-Krieger, S. Theory of Plates and Shells; McGraw-Hill: New York, 1959. 